\title{
Urinary kidney injury molecule-1 as an early indicator to predict contrast-induced acute kidney injury in patients with diabetes mellitus undergoing percutaneous coronary intervention
}

\author{
WENHUA LI* ${ }^{*}$ YAREN YU*, HAIYAN HE* , JING CHEN and DEBIN ZHANG \\ Department of Cardiology, Affiliated Hospital of Xuzhou Medical College, Xuzhou, Jiangsu 221002, P.R. China
}

Received January 20, 2015; Accepted March 20, 2015

DOI: $10.3892 /$ br.2015.449

\begin{abstract}
With the improvement of the skill level of coronary intervention, contrast agents are used more widely. As a result, contrast-induced acute kidney injury (CI-AKI) is currently the third leading cause of hospital-acquired AKI. Traditionally, AKI is defined by measuring an increase of the serum creatinine concentration (Scr). CI-AKI indicates impairment in renal function, which is diagnosed as an elevation in the $\mathrm{SCr}$ levels following intravascular injection of the contrast media. However, Scr is an insensitive indicator for detecting CI-AKI. The present study was designed to investigate whether human urinary kidney injury molecule-1 (KIM-1) is an early marker to predict CI-AKI in patients with diabetes mellitus undergoing percutaneous coronary intervention (PCI). The present study includes the general clinical data of 145 patients with diabetes mellitus who underwent PCI between March 1, 2013 and December 31, 2013. A non-ionic, low osmolarity contrast agent was used during the present study. The Scr levels and estimated glomerular filtration rate were measured prior to and within 24 and $48 \mathrm{~h}$ after the injection of contrast agents. Urinary samples were collected prior to and within 2, 6, 12, 24 and $48 \mathrm{~h}$ after the coronary interventional procedure. Simultaneously, the urinary KIM-1 values were measured using an ELISA kit. CI-AKI was diagnosed as an increase of $\geq 0.5 \mathrm{mg} / \mathrm{dl}$ or $\geq 25 \%$ in Scr concentration over baseline, 24-48 h after the procedure. In total, 19 of 145 (13.1\%) patients exhibited CI-AKI. There was a significant difference $(\mathrm{P}<0.05)$ between the urinary KIM-1 levels measured 2, 6, 12, 24 and $48 \mathrm{~h}$ after the procedure and those prior to the procedure in the CI-AKI group. There was no significant difference between the Scr values measured $24 \mathrm{~h}$ after the procedure and
\end{abstract}

Correspondence to: Professor Wenhua Li, Department of Cardiology, Affiliated Hospital of Xuzhou Medical College, 99 Huaihai West Road, Xuzhou, Jiangsu 221002, P.R. China E-mail: xzwenhua0202@163.com

*Contributed equally

Key words: kidney injury molecule-1, percutaneous coronary intervention, contrast-induced acute kidney injury those prior to the procedure. Evidently, using KIM-1 values to predict CI-AKI was $<24 \mathrm{~h}$ earlier compared to using Scr values. The area under the receiver operating characteristic curve of KIM-1 $24 \mathrm{~h}$ after the procedure was 0.856 and the $95 \%$ confidence interval of the corresponding area was 0.782-0.929. When the pivotal point of CI-AKI diagnosis was $6,327.755 \mathrm{pg} / \mathrm{ml}$, the specificity was $85.7 \%$ and the sensitivity was $73.7 \%$. Univariate analysis showed that the Scr concentration was positively correlated with the urinary KIM-1 level during the time prior to the procedure and 24 and $48 \mathrm{~h}$ after the procedure. In conclusion, the urinary KIM-1 may be a potential indicator for the early diagnosis of CI-AKI.

\section{Introduction}

The contrast-induced acute kidney injury (CI-AKI) has become the third leading cause of hospital-acquired acute kidney injury (AKI) (1). Traditionally, AKI is defined by measuring an increase of the serum creatinine concentration (Scr). Elevated levels of SCr following intravascular injection of the contrast media indicates impairment of renal function patients with CI-AKI $(2,3)$. With the increasing number of patients who received coronary intervention procedure therapy, the morbidity and mortality rate of CI-AKI has increased, particularly in those patients with diabetes mellitus and chronic kidney disease. However, $<30 \%$ of patients who underwent percutaneous coronary intervention (PCI) had diabetes mellitus, and suffered from a higher risk of CI-AKI than those without diabetes mellitus. Therefore, it is crucial to make an early diagnosis and prognosis of CI-AKI in patients with diabetes mellitus. However, using the Scr levels as the AKI indicator has numerous defects owing to that fact that it may be affected by a number of non-renal factors, including ethnicity, age, basic metabolism and nutrition. Only when the estimated glomerular filtration rate (eGFR) level has decreased to $50 \%$ of the normal level can the Scr level be increased $(4,5)$.

A previous study has shown that KIM-1 is a transmembrane glycoprotein located in the membrane of renal proximal tubule epithelial cell. There was little expression in the human normal renal tissue, but an increased expression evidently occurred when the renal tissue suffered ischemia and hypoxia. Furthermore, it was positively correlated with the severity of renal injury (6). The expression levels of KIM-1 in urine were consistent with the levels in renal tissue. Currently, a 
large number of experimental evidence has suggested that KIM-1 is not only a good biological indicator of acute kidney injury, but also as a functional molecule to participate in the process of renal tubular damage and repair (7). The levels of KIM-1 in renal tissue measured $2 \mathrm{~h}$ after injection of contrast agents evidently increased, however, until $12 \mathrm{~h}$ after injection of contrast agents, the injury of renal tubular epithelial cells was shown to occur in the renal tissue biopsy, which has been shown in a previous rat model of low permeability, contrast agent-induced acute injury of renal tubular epithelial cell. The present study was designed to confirm whether urinary KIM-1 is an earlier diagnosis and prognosis indicator compared to Scr in CI-AKI patients with diabetes mellitus who underwent PCI.

\section{Patients and methods}

Patient population. The study includes the general clinical data of 145 patients with diabetes mellitus who underwent PCI procedures at the Department of Cardiology, Affiliated Hospital of Xuzhou Medical College (Xuzhou, China) between March 1, 2013 and December 31, 2013. In total, 54 were female and 91 were male, and the average age was $66.8 \pm 9.9$ years. Exclusion criteria were: i) Severe hepatic and renal dysfunction; ii) the use of drugs with renal injury during the preoperative period; iii) severe heart failure or left ventricular ejection fraction $<35 \%$; iv) tumors; v) acute or chronic infectious diseases; vi) thyroid or adrenal dysfunction; and vii) pregnant or breast-feeding women. The osmotic concentration was $800 \mathrm{mOsm} / \mathrm{kg}$. All the patients routinely received anticoagulation, antiplatelet, antiangina and conventional hydration therapy, as well as monitoring of blood pressure, lipids and blood glucose.

The study was approved by the Ethics Committee of the Affiliated Hospital of Xuzhou Medical College. All the patients provided written informed consent for the procedure.

Laboratory assay. SCr and other corresponding indicators were measured. Fasting blood specimens were collected prior to and 24 and $48 \mathrm{~h}$ after the procedure in the biochemical laboratory and were subsequently analyzed using an Olympus AU2700 Automatic Biochemistry Analyzer (Olympus, Center Valley, PA, USA) for determination. Urine specimens were collected prior to and at 2, 6, 12,24 and $48 \mathrm{~h}$ after the procedure and were immediately centrifuged $\left(1,409 \mathrm{x}\right.$ g for $20 \mathrm{~min}$ at $\left.4^{\circ} \mathrm{C}\right)$. The superficial fractions were collected and stored at $-80^{\circ} \mathrm{C}$. The levels of urinary KIM-1 were measured using an ELISA kit purchased from R\&D Systems (Minneapolis, MN, USA). Renal function was calculated by the eGFR using the Modification of Diet in Renal Disease formula for Chinese patients (8): GFR $\left(\mathrm{ml} / \mathrm{min} / 1.73 \mathrm{~m}^{2}\right)=175 \times \mathrm{SCr}(\mathrm{mg} / \mathrm{dl})^{-1.1549} \mathrm{x} \mathrm{age}^{-0.2039}(\mathrm{x} 0.79$ if female). CI-AKI was diagnosed as an increase of $\geq 0.5 \mathrm{mg} / \mathrm{dl}$ or $\geq 25 \%$ in SCr levels over the baseline $24-48 \mathrm{~h}$ after the intravascular injection of contrast medium, without an alternative etiology (9).

Statistical analysis. Continuous variables are expressed as the mean \pm standard deviation. The Student's t-test and one-way analysis of variance were used for the comparison of continuous variables. Categorical data are shown as absolute values and percentages. The $\chi^{2}$ or the Fisher's exact test were used for
Table I. Differences in the SCr and eGFR values prior and subsequent to the procedure in the two groups.

\begin{tabular}{lcc}
\hline Groups & $\mathrm{SCr}, \mu \mathrm{mol} / \mathrm{l}$ & $\mathrm{eGFR}, \mathrm{ml} / \mathrm{min}^{-1} / 1.73 \mathrm{~m}^{2}$ \\
\hline $\begin{array}{l}\text { No-CI-AKI } \\
\text { Pre-procedure }\end{array}$ & $60.41 \pm 14.20$ & $109.42 \pm 23.72$ \\
Post-procedure & & \\
24 h & $66.87 \pm 16.01$ & $105.86 \pm 25.58$ \\
$48 \mathrm{~h}$ & $66.54 \pm 16.80$ & $106.94 \pm 26.16$ \\
CI-AKI & & \\
Pre-procedure & $70.58 \pm 9.97$ & $97.00 \pm 16.93$ \\
Post-procedure & & \\
$24 \mathrm{~h}$ & $74.05 \pm 12.58$ & $88.48 \pm 16.54^{\mathrm{a}}$ \\
$48 \mathrm{~h}$ & $92.32 \pm 15.05^{\mathrm{a}}$ & $68.69 \pm 14.19^{\mathrm{a}}$ \\
\hline
\end{tabular}

${ }^{\text {a }} \mathrm{P}<0.05$, compared to the non-CI-AKI group. Data are presented as the mean \pm standard deviation. SCr, serum creatinine; eGFR, estimated glomerular filtration rate; CI-AKI, contrast-induced acute kidney injury.

the comparison of categorical variables. The Pearson's correlation analysis was used to evaluate the correlations. $\mathrm{P}<0.05$ was considered to indicate a statistically significant difference. All the hypothesis testing was two-tailed. The SPSS version 16.0 (SPSS, Inc., Chicago, IL, USA) package was used for all calculations.

\section{Results}

Differences in SCr and $G F R$ values prior and subsequent to the procedure. The differences in $\mathrm{SCr}$ and $\mathrm{eGFR}$ values are shown in Table I. Samples were collected from all 145 patients with diabetes mellitus undergoing PCI. In total, 19 of 145 (13.1\%) patients were diagnosed with CI-AKI. The 145 patients were categorized to a CI-AKI (19 patients) and non-CI-AKI group (126 patients). No significant difference was shown in gender, age, hemoglobin, body mass index, eGFR, SCr and the incident rate of hypertension between the CI-AKI and non-CI-AKI groups. The therapy and hydration volumes used were also not statistically different during hospitalization. The peak level of Scr in the CI-AKI group $(92.32 \pm 15.05 \mu \mathrm{mol} / \mathrm{l})$ was clearly higher than that in the non-CI-AKI group $(66.54 \pm 16.80 \mu \mathrm{mol} / \mathrm{l})$ at $48 \mathrm{~h}$ after the procedure $(\mathrm{P}<0.05)$. There was a significant difference $(\mathrm{P}<0.05)$ in the level of eGFR at 24 and $48 \mathrm{~h}$ after the procedure between the non-CI-AKI groups and CI-AKI groups.

Differences in the level of urinary KIM-1 prior and subsequent to the procedure. The differences in the level of urinary KIM-1 are shown in Table II. The urinary KIM-1 levels were increased $2 \mathrm{~h}$ after the procedure in the non-CI-AKI group, but no statistically significant difference was identified $(\mathrm{P}>0.05)$. There was a significant difference $(\mathrm{P}<0.05)$ between the urinary KIM-1 levels measured 2, 6, 12, 24 and $48 \mathrm{~h}$ after the procedure and those before the procedure in the CI-AKI group, and for the levels measured $6,12,24$ and $48 \mathrm{~h}$ after the procedure in non-CI-AKI groups. Furthermore, significant differences in the 
Table II. Differences in the urinary KIM-1 prior and subsequent to the procedure in the two groups.

\begin{tabular}{lcc}
\hline & \multicolumn{2}{c}{ Urinary KIM-1, pg/ml } \\
\cline { 2 - 3 } Procedure & Non-CI-AKI & CI-AKI \\
\hline Pre & $3,863.07 \pm 1,081.56$ & $3,515.98 \pm 945.58$ \\
Post & & \\
2 & $4,037.53 \pm 1,148.11$ & $4,015.84 \pm 855.96^{\mathrm{b}}$ \\
6 & $4,370.46 \pm 1,278.46^{\mathrm{a}}$ & $5,095.32 \pm 1,325.09^{\mathrm{b}, \mathrm{c}}$ \\
12 & $4,619.10 \pm 1,379.54^{\mathrm{b}}$ & $5,982.04 \pm 1,506.62^{\mathrm{b}, \mathrm{d}}$ \\
24 & $4,858.56 \pm 1,490.54^{\mathrm{b}}$ & $6,984.20 \pm 1,441.87^{\mathrm{b}, \mathrm{d}}$ \\
48 & $4,663.49 \pm 1,399.67^{\mathrm{b}}$ & $6,078.81 \pm 1,519.56^{\mathrm{b}, \mathrm{d}}$ \\
\hline
\end{tabular}

${ }^{\mathrm{a}} \mathrm{P}<0.05,{ }^{\mathrm{b}} \mathrm{P}<0.01$, compared to the pre-procedure. ${ }^{\mathrm{c}} \mathrm{P}<0.05,{ }^{\mathrm{d}} \mathrm{P}<0.01$, compared to the non-CI-AKI group. Data are presented as the mean \pm standard deviation. KIM-1, kidney injury molecule-1; CI-AKI, contrast-induced acute kidney injury.

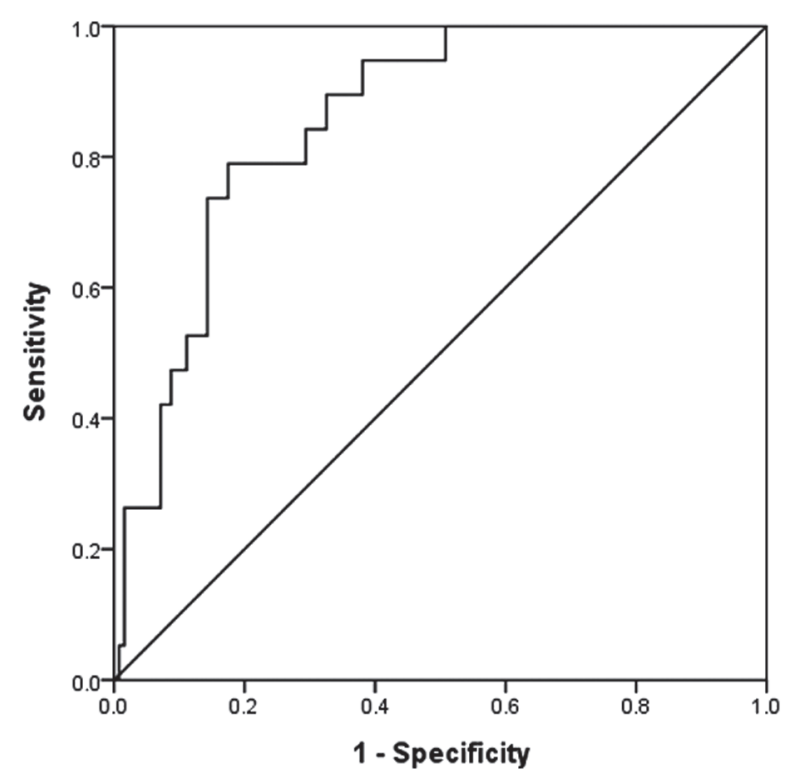

Figure 1. ROC analysis shows a high area under the curve for the $24 \mathrm{~h}$ postoperative urinary KIM-1. At a cut-off level of $>6,327.755 \mathrm{pg} / \mathrm{ml}$, urinary KIM-1 exhibited $73.7 \%$ sensitivity and $85.7 \%$ specificity for detecting CI-AKI. Area under the ROC curve, 0.856; 95\% confidence interval, 0.782-0.929; $\mathrm{P}<0.05$ ROC, receiver operating characteristic; KIM-1, kidney injury molecule-1; CI-AKI, contrast-induced acute kidney injury.

urinary KIM-1 levels measured 6, 12, 24 and $48 \mathrm{~h}$ were identified compared to those in the non-CI-AKI. The KIM-1 levels had not returned to the normal level $48 \mathrm{~h}$ after the procedure.

Receiver operating characteristic (ROC) curve analysis. The ROC curve for the level of urinary KIM-1 measured $24 \mathrm{~h}$ after the procedure is shown in Fig. 1. The area under the ROC curve of KIM-1 $24 \mathrm{~h}$ after the procedure was 0.865 and the $95 \%$ confidence interval of the corresponding area was 0.782-0.929. When the pivotal point of diagnosis of CI-AKI was $6,327.755 \mathrm{pg} / \mathrm{ml}$, the specificity was $85.7 \%$ and the sensitivity was $73.7 \%$.
Correlation between SCr and urinary KIM-1 concentration. Bivariate analysis showed that the level of urinary KIM-1 measured prior to and $24-48 \mathrm{~h}$ after the procedure positively correlated with $\mathrm{SCr}$ at the identical time points. Preoperative $\mathrm{SCr}$ and preoperative urinary KIM-1 ( $\mathrm{r}=0.322, \mathrm{P}<0.01)$, postoperative $24 \mathrm{~h} \mathrm{SCr}$ and postprocedure $24 \mathrm{~h}$ urine KIM-1 ( $\mathrm{r}=0.317, \mathrm{P}<0.01$ ), and postoperative $48 \mathrm{~h} \mathrm{SCr}$ and postprocedure $48 \mathrm{~h}$ urinary KIM-1 $(\mathrm{r}=0.453, \mathrm{P}<0.01)$ showed positive correlations.

\section{Discussion}

With the development of interventional diagnosis and treatment technology in recent years, an increasing number of patients with coronary disease prefer accepting interventional treatment owing to its numerous advantages, such as simple surgery, less pain, low risk and fast postoperative recovery rather than surgical therapy. Approximately $30 \%$ of patients with coronary disease have suffered from diabetes mellitus, and they may have severe conditions predisposing them to CI-AKI (10). CI-AKI has become one of the three major reasons of deteriorating the prognosis of the patients after PCI (11). Due to this, CI-AKI has received extensive attention from medical experts. However, there is a lack of effective measures to control and prevent CI-AKI at present. Therefore, early diagnosis and treatment of CI-AKI has played a crucial role in patients with diabetes mellitus undergoing PCI. Traditionally, it is defined by measuring the elevation of the Scr concentration, but is currently considered a poor indicator of acute renal dysfunction (12). Only when the eGFR decreases to $<50 \%$ of the normal rate is the $\mathrm{SCr}$ level likely to increase. Therefore, the early diagnosis of CI-AKI is critical for prevention (13).

It is generally accepted that the pivotal reason for causing CI-AKI is renal medulla hypoxic (14), which is initiated by three different ways: Contrast agent-induced hemodynamic changes, oxygen free radical damage and a direct toxic effect to renal tubular disease (15). KIM-1 is a transmembrane glycoprotein located in the membrane of renal proximal tubule epithelial cells. There is little expression in the human normal renal tissue, but an increased expression evidently occurred when the renal tissue was injured (6). A previous study has shown that KIM-1 may participate in the process of renal tubular damage and repair, which has a protective effect on acute kidney injury and an adverse effect on chronic kidney disease (7). The expression levels of KIM-1 in urine were consistent with the levels in renal tissue $(16,17)$. As previously described, urinary KIM-1 is regarded as an early biomarker of acute renal tubular injury owing to its high sensitivity and specificity for predicting ischemic renal injury, and it also has an ability to distinguish between acute ischemic renal injury and chronic kidney insufficiency (11). Using urinary KIM-1 to detect various factors causing renal injury was much earlier than using other traditional biomarkers, such as Scr, urea nitrogen, urine sugar and urine protein. Ichimura et al (18) showed that the expression levels of KIM-1 following renal injury clearly increased, and it occurred earlier than the levels of Scr. The present study confirmed that urinary KIM-1 could be considered as an improved early diagnostic indicator for CI-AKI. 
The result of the correlation analysis has shown positive correlations between the urinary KIM-1 concentration and $\mathrm{SCr}$ levels measured pre- and postprocedure in the present study. In the CI-AKI group, the preoperative value was statistically significantly different compared to the level of Scr $48 \mathrm{~h}$ after the procedure, the eGFR value was significantly different at $24 \mathrm{~h}$ after the procedure, while the urinary KIM-1 level differed $2 \mathrm{~h}$ after the procedure. In summary, the urinary KIM-1 level rises earlier than SCr in CI-AKI, which also agreed with the results of the study by Ichimura et al (18). The only effective prevention measure for CI-AKI is universally acknowledged as hydration therapy (19).

Urinary KIM-1 can be measured using specific ELISAs, which are reliable, fast and economical, to test urine specimens from patients. This is a non-invasive and convenient technique, which therefore can be extended clinically. In addition, postoperative urinary KIM-1 offers an early prediction of the emergence of CI-AKI, and may provide early intervention measures to benefit patients. Therefore, KIM-1 was a better indicator for the early prediction of CI-AKI.

The small sample size and a variety of confounding factors are the limitations of the present study. Further studies that are characterized by larger sample sizes, with a controlled group of patients without diabetes mellitus and identify and exclude potential factors that may also cause increases in KIM-1 levels are required to confirm the findings of the present study.

\section{References}

1. Caixeta A and Mehran R. Evidence-based management of patients undergoing PCI: contrast-induced acute kidney injury. Catheter Cardiovasc Interv 75 (Suppl 1): S15-S20, 2010.

2. Parfrey P: The clinical epidemiology of contrast-induced nephropathy. Cardiovasc Intervent Radiol 28 (Suppl 2): S3-S11, 2005.

3. Mehran R and Nikolsky E: Contrast-induced nephropathy: definition, epidemiology, and patients at risk. Kidney Int Suppl (100): S11-S15, 2006.
4. Odutayo A and Cherney D: Cystatin C and acute changes in glomerular filtration rate. Clin Nephrol 78: 64-75, 2012.

5. Mehran R: Contrast-induced nephropathy remains a serious complication of PCI. J Interv Cardiol 20: 236-240, 2007.

6. Tonomura Y, Tsuchiya N, Torii M and Uehara T: Evaluation of the usefulness of urinary biomarkers for nephrotoxicity in rats. Toxicology 273: 53-59, 2010.

7. Huo W, Zhang K, Nie Z, Li Q and Jin F: Kidney injury molecule-1 (KIM-1): A novel kidney-specific injury molecule playing potential double-edged functions in kidney injury. Transplant Rev (Orlando) 24: 143-146, 2010.

8. Malhis M, Al-Bitar S and Al-Deen Zaiat K: The role of theophylline in prevention of radiocontrast media-induced nephropathy. Saudi J Kidney Dis Transpl 21: 276-283, 2010.

9. McCullough PA and Soman SS: Contrast-induced nephropathy. Crit Care Clin 21: 261-280, 2005.

10. Shaw JE, Sicree RA and Zimmet PZ: Global estimates of the prevalence of diabetes for 2010 and 2030. Diabetes Res Clin Pract 87: 4-14, 2010.

11. Han W K, Bailly V, Abichandani R, Thadhani R and Bonventre JV: Kidney Injury Molecule-1 (KIM-1): A novel biomarker for human renal proximal tubule injury. Kidney Int 62: 237-244, 2002.

12. Parikh CR, Abraham E, Ancukiewicz M and Edelstein CL: Urine IL-18 is an early diagnostic marker for acute kidney injury and predicts mortality in the intensive care unit. J Am Soc Nephrol 16: 3046-3052, 2005.

13. Melnikov VY, Ecder T, Fantuzzi G, et al: Impaired IL-18 processing protects caspase-1-deficient mice from ischemic acute renal failure. J Clin Invest 107: 1145-1152, 2001.

14. Heyman S N, Rosen S and Rosenberger C: Renal parenchymal hypoxia, hypoxia adaptation, and the pathogenesis of radiocontrast nephropathy. Clin J Am Soc Nephrol 3: 288-296, 2008.

15. Katzberg RW: Contrast medium-induced nephrotoxicity: Which pathway? Radiology 235: 752-755, 2005.

16. Bailly V, Zhang Z, Meier W, et al: Shedding of kidney injury molecule-1, a putative adhesion protein involved in renal regeneration. J Biol Chem 277: 39739-39748, 2002.

17. Vaidya VS, Ramirez V, Ichimura T, et al: Urinary kidney injury molecule-1: A sensitive quantitative biomarker for early detection of kidney tubular injury. Am J Physiol Renal Physiol 290: F517-F529, 2006.

18. Ichimura $\mathrm{T}$, Bonventre $\mathrm{J} \mathrm{V}$, Bailly $\mathrm{V}$, et al: Kidney injury molecule-1 (KIM-1), a putative epithelial cell adhesion molecule containing a novel immunoglobulin domain, is up-regulated in renal cells after injury. J Biol Chem 273: 4135-4142, 1998.

19. Thomsen HS: How to avoid CIN: guidelines from the European Society of Urogenital Radiology. Nephrol Dial Transplant 20 (Suppl 1): i18- i22, 2005. 